\title{
DIFFERENCE BETWEEN Be STARS AND SHELL STARS FROM H $\alpha$ EMISSION
}

\author{
H. CHEN and A. R. TAYLOR \\ Department of Physics and Astronomy \\ University of Calgary, Canada
}

\begin{abstract}
We examine $\mathrm{H} \alpha$ equivalent widths versus spectral-types for $41 \mathrm{Be}$ stars. Although the $W_{\alpha}$ value is scattered for a given spectral-type, a well-defined upper limit exists. Most shell stars are located in the upper limit region. The shell stars all have $v \sin i / v_{\text {cri }}$ larger than the normal Be stars in the upper limit region. This strongly suggests that the distinction between shell stars and normal Be stars of high equivalent width is a result of variation in inclination angle $i$. Polarization data also support this hypothesis.
\end{abstract}

\section{Introduction}

What causes the difference between a "normal" Be star and a shell star is still not clearly understood. On one hand, Hutchings (1976) suggested that "A shell may however be simply a very extended Be star envelope." On the other hand, Slettebak et al. (1992) recently claim that shell stars have envelopes which are more compact and have lower electron densities than Be stars, based on their spectroscopic observations of 41 bright stars.

In this investigation we re-examine the data of Slettebak et al. (1992) to see if there is any difference between shell stars and "normal" Be stars in terms of their $W_{\alpha}$ values.

\section{Results}

In Fig. 1, we plot $W_{\alpha}$ versus spectral types for 41 stars using Slettebak et al. data. Although $W_{\alpha}$ value is scattered for a given spectral-type, a welldefined upper limit exists. This upper limit peaks at about B2 and decreases towards later-types. A similar plot for a larger number of Be stars suggests that this upper limit might also decrease towards earlier-types (Chen \& Talyor 1993). With one exception, the shell stars are located in the upper limit region (above the dash line). As a group, they have higher equivalent widths than "normal" Be stars with same spectral type. The only exception is $\zeta$ Tau, which is in a binary system (Slettebak, 1982).

In the upper limit region, there are also stars which show "normal" Be star spectra. It is noteworthy that the shell stars in this region all have $v$ $\sin i / v_{c r i}$ larger than the normal Be stars (Fig. 2). This suggests that the distinction between shell stars and normal Be stars of high equivalent width is a result of variation in $\sin i$ or $v / v_{c r i}$. If all these stars are rotating at a high speed, one can conclude normal Be stars have smaller inclination angles 


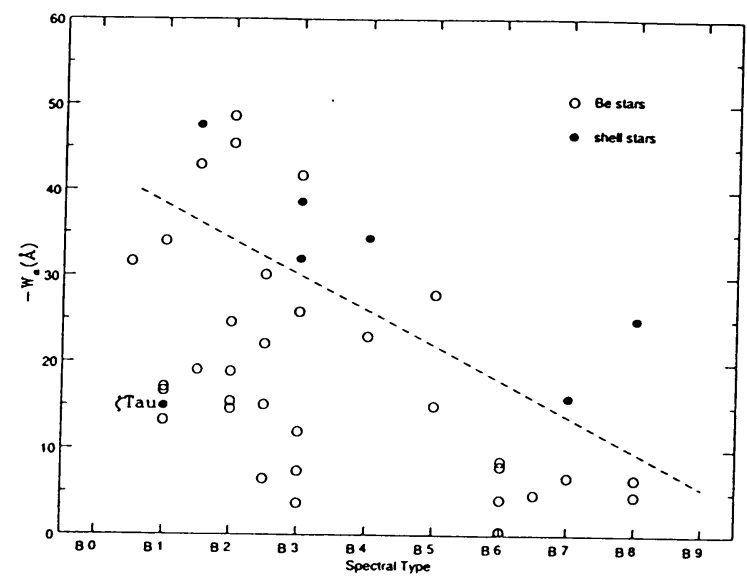

Fig. 1. $W_{\alpha}$ versus spectral types

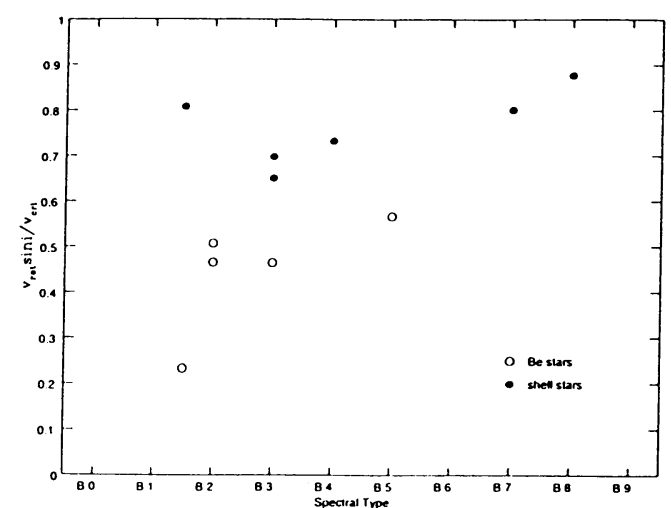

Fig. 2. The values of $v \sin i / v_{c r i}$ for the stars above the dash line in Fig. 1

than shell stars. Of seven shell stars and five normal Be stars above the dash line in Fig. 1, intrinsic polarization data are available for six shell stars and three normal Be stars (McLean \& Brown 1978). All shell stars have higher intrinsic polarization than normal Be stars of the same spectral types. This again supports the hypothesis that the shell stars have higher inclination angles than the normal Be stars of high $W_{\alpha}$.

\section{The References}

Chen, H. \& Taylor, A. R., in preparation, (1993).

Huchings, J. B., in IAU Symp. 70, Be and Shell Stars, ed. A. Slettebak (Sordrecht:Reidel), 13 (1976).

McLean, I. S., \& Brown, J. C., Astr. Astrophys. 69, 291 (1978).

Slettebak, A., Astrophys. J. Suppl., 50, 55 (1982).

Slettebak, A., Collins II, G.W., \&Truax, R., Astrophys. J. Suppl. 81, 335 (1992). 